(C) 2019 IEEE. Personal use of this material is permitted. Permission from IEEE must be obtained for all other uses, in any current or future media, including reprinting/republishing this material for advertising or promotional purposes, creating new collective works, for resale or redistribution to servers or lists, or reuse of any copyrighted component of this work in other works. 


\title{
Deep Learning-Based Neural Network Training for State Estimation Enhancement: Application to Attitude Estimation
}

\author{
Mohammad Al-Sharman, Yahya Zweiri, Mohammad A. Jaradat, Raghad Al-Husari, Dongming \\ Gan and Lakmal Seneviratne
}

\begin{abstract}
Achieving precise state estimation is needed for the unmanned aerial vehicle to perform a successful flight with a high degree of stability. Nonetheless, obtaining accurate state estimation is considered challenging due to the inaccuracies associated with the measurements of the onboard commercial-offthe-shelf (COTS) Inertial Measurement Unit (IMU). The immense vibration of the vehicle's rotors makes these measurements suffer from issues like; large drifts, biases and immense unpredictable noise sequences. These issues cannot be significantly tackled using classical estimators and an accurate sensor fusion technique needs to be developed. In this paper, a deep learning framework is developed to enhance the performance of the state estimator. A deep neural network (DNN) is trained using a deep-learning-based technique to identify the associated measurement noise models and filter them out. Dropout technique is adopted for training the DNN to avoid overfitting and reduce the complexity of nets computations. Compared to the classical estimation results, the proposed deep learning technique demonstrates capabilities in identifying the measurement's noise characteristics. As an example, an enhancement in estimating the attitude states at near hover is proved using this approach. Furthermore, an actual hover flight was performed to validate the proposed estimation enhancement method.
\end{abstract}

Index Terms-Multirotor UAV; Attitude Determniation; Deep Learning; Dropout method.

\section{INTRODUCTION}

$\mathrm{R}$ OTARY UAVs (RUAVs), such as single and multirotor vehicles, are yet preferred in missions that require precise execution [1, 2, 3]. In addition to their small size and lightweight, their remarkable capabilities in performing hovering, vertical takeoff and landing make them unique candidates in many civil and military applications. RUAVs are

Mohammad Al-Sharman is with the Department of Electrical and Computer engineering, University of Waterloo, ON, Canada (e-mail: mkalsharman@uwaterloo.ca).

Yahya Zweiri is with the Faculty of Science, Engineering and Computing, Kingston University London, London SW15 3DW, UK and Khalifa University Center for Autonomous Robotic System, Khalifa University, P.O. Box 127788, Abu Dhabi, UAE (e-mail: y.zweiri@kingston.ac.uk).

M. A. Jaradat is with the Department of Mechanical Engineering, the American University of Sharjah, Sharjah, UAE (e-mail: mjaradat@aus.edu)/ Jordan University of Science \& technology, Irbid, Jordan.

Raghad Al husari is with Khalifa University Center for Autonomous Robotic System, Khalifa University of Science and Technology, Abu Dhabi, UAE (email: raghad.husari@ku.ac.ae).

Dongming Gan is with the Department of Mechanical Engineering, Khalifa University of Science and Technology, Abu Dhabi, UAE (e-mail: dongming.gan@ku.ac.ae).

Lakmal Seneviratne is with the Khalifa University Center for Autonomous Robotic Systems, P.O. Box 127788, Abu Dhabi, UAE (e-mail: lakmal.seneviratne@ku.ac.ae). recently employed in several sets of applications, such as power line inspection [4], trajectory planning [5] and gripping and robotics applications [6]. Several problems are associated with the attitude determination and control have been extensively studied to enhance the robustness of the autonomous performance of the vehicle $[7,8]$.

Precise attitude estimation is essential for the RUAVs to boost their agility and maneuverability [9]. However, developing an accurate attitude estimation technique for such highly nonlinear dynamic systems is not a straightforward problem due to some shortcomings related to the measurements of the onboard low cost sensors $[10,11]$. These off-the-shelf measurements suffer from the large amount of biases, drifts and immense noise sequences. These problems have been recently tackled by developing sensor fusion techniques, such as the Kalman-based state estimation techniques [12, 13]. Integrating such techniques with highly nonlinear systems, such as RUAVs, to improve their attitude estimation and control has gained a great attention in the last few years [12]. For instance, the conventional linear Kalman filter is used to resolve the problem of the attitude estimation of the Maxi Joker flaybarless helicopter [14]. The nonlinear extended Kalman filter (EKF) and the Unscented Kalman (UKF) filters have been also utilized to deal with nonlinearities of the RUAV's systems [15, 16, 17].

Incorporating intelligent algorithms with the sensor fusion architectures has been extensively studied in the last few years [18]. Several recent published works have proposed intelligent sensor fusion approaches using fuzzy logic, neural networks and genetic algorithms $[19,20]$. A fuzzy logic is integrated with the conventional Kalman to reduce the measurement noise and enhance the position estimation of capacitive touch panels [21]. Similarly, an adaptive multi-sensor fusion technique has been implemented to perform a precision landing for the RUAV system [22]. The fuzzy rules have been implemented to adapt the variances of the measurement covariance matrix $R$ of the Kalman filter. Furthermore, the hybrid intelligent systems have been used for state estimation approaches. A hybrid NeuroFuzzy system has been used to enhance the Kalman attitude estimation [23]. Using neural networks, an intelligent sensor fusion technique has been design to enhance attitude estimation of the low cost GPS/INS navigation system [24]. The aforementioned intelligent sensor fusion approaches prove their superiority in rejecting the measurement noise and providing more accurate estimates.

Deep learning (DL) is defined as a machine learning technique that employs the multi-layer neural network which consists of two or more hidden layers [25]. DL algorithms with multi-layer neural networks have exhibited an excellent 
performance in applications related to, image processing and features detection, objects identifications and speech transcription [26, 27, 28, 29]. In addition, DL has demonstrated its applicability to control dynamic systems. The DL methods have been employed to replace the classical PID controller of the used control scheme [30]. In UAV's applications, DL has been implemented to perform several missions. Table 1 presents a list of example application of using DL in UAV navigational missions [31]. From table 1, it can be seen that DL has been utilized in UAV's for trajectory planning, motion control and for image processing applications.

The published work in the field of DL in UAVs has focused on developing DL framework for certain UAV application rather than enhancing the performance of the UAV maneuverability itself. However, implementing the DL approaches to improve the robustness of the UAV control and state estimation techniques has started receiving an attention. For instance, DL has been introduced to resolve the problem vision-based attitude rate estimation using Convolutional Neural Networks (CNN) [32]. The solution is computationally heavy and might cause delays as images from 3 cameras have to be processed to estimate the UAV's orientation. Moreover, it burdens the UAV by the extra weight of vision sensors.

Exploring the connections between deep learning architectures and recurrent state estimation has been getting an attention from researchers in the field. For instance, deep Kalman filters have proved its capability in state estimation and noise rejection [33]. In this paper, a novel DL technique has been developed to identify the associated measurement's noise characteristics which results in state estimation enhancement. A DNN has been trained using the recent innovative DL method which is called dropout [34]. This learning method has been used to resolve the overfitting problem of the back propagation while training the network. In addition, dropout learning method has demonstrated an ability in providing robust representations for the model uncertainty and the abstractions in data [35]. Exploiting these advantages to enhance the estimator's capability of rejecting the immense noise is the main focus of this research. Dropout learning method has been implemented to train the 3-hidden-layers DNN to identify the unfiltered Gaussian noise associated with Kalman attitude estimates. After identifying the noise models, the DNN has

Table 1: Deep learning techniques for UAV applications

\begin{tabular}{|c|c|c|c|c|c|}
\hline References & $\begin{array}{c}\text { UAV } \\
\text { Platform }\end{array}$ & DL Approach & Purpose & $\begin{array}{c}\text { Sensing } \\
\text { Technique }\end{array}$ & Method \\
\hline [45] & Generic & DNN & $\begin{array}{l}\text { Navigation for } \\
\text { Power line } \\
\text { inspection. }\end{array}$ & Images & $\begin{array}{c}\text { - DL for processing images that } \\
\text { captured by the onboard optical } \\
\text { sensor. }\end{array}$ \\
\hline [46] & Generic & RNN & Trajectory planning & $\begin{array}{l}\text { UAV's } \\
\text { location }\end{array}$ & $\begin{array}{l}\text { - RNN for designing a handover } \\
\text { algorithm for UAV network. }\end{array}$ \\
\hline [47] & $\begin{array}{l}\text { Bebop } 2 \\
\text { micro } \\
\text { RUAV }\end{array}$ & DNN & Motion control & Images & $\begin{array}{l}\text { - DNN for UAV control based on } \\
\text { human gestures. }\end{array}$ \\
\hline $\begin{array}{l}{[48]} \\
{[49]}\end{array}$ & RUAV & & & & $\begin{array}{l}\text { - Processing optical images of the } \\
\text { scene. } \\
\text { - Processing Tobacco planting } \\
\text { images taken by UAV onboard } \\
\text { camera. }\end{array}$ \\
\hline$[50]$ & Generic & & & & $\begin{array}{l}\text { - Detecting target vehicles and } \\
\text { monitoring the traffic flow. }\end{array}$ \\
\hline$[51]$ & RUAV & & & & $\begin{array}{l}\text { - Estimating the center of the gates } \\
\text { during drone racing. }\end{array}$ \\
\hline $\begin{array}{l}{[52]} \\
{[53]} \\
{[54]}\end{array}$ & $\begin{array}{l}\text { Generic } \\
\text { Generic } \\
\text { Generic }\end{array}$ & CNN & $\begin{array}{l}\text { Object recognition } \\
\text { and feature } \\
\text { extraction. }\end{array}$ & $\begin{array}{l}\text { UAV } \\
\text { Imagery }\end{array}$ & $\begin{array}{l}\text { - Multi-labeling UAV Imagery. } \\
\text { - Detecting cars for UAV Imagery. } \\
\text { - Detecting moving targets for } \\
\text { UAV. }\end{array}$ \\
\hline$[56]$ & 3DR Iris+ & & & & $\begin{array}{l}\text { - Extracting parking lots from the } \\
\text { images taken by UAV optical. } \\
\text { sensors. } \\
\text { - Determining MAV's orientation } \\
\text { and lateral offset for trail } \\
\text { following. }\end{array}$ \\
\hline
\end{tabular}


been trained to reject these noise sequences and provides values close to the real ones.

The proposed method is also practical in real applications where inexpensive sensors are used for precision missions. To the extent of the authors' knowledge and after surveying the literature, incorporating the Dropout techniques to enhance the classical estimation technique has not been introduced before. The rest of the paper is structured as follows Section II describes the multirotor attitude. Section III presents the proposed DLNN attitude estimation framework. In Section IV, the simulation results of the attitude states are introduced. Section V presents the results of the experimental validation of the proposed approach. Finally, Section VI concludes the paper.

\section{BACKGROUND}

In this section, we review the formulation of the multirotor attitude dynamics and the main structure of the multilayer FeedForward Neural Network (FFNN).

\section{A. Multirotor Attitude Dynamics}

Multirotor platforms are preferred in several civil and military application s. It is sustained in the air by the lift of the rotors. The dynamics of the vehicle orientation can be described by the Euler angles $\left(\Theta=\left[\begin{array}{lll}\phi & \theta & \psi\end{array}\right]^{T}\right)$ which represent the orientation of the B-frame with respect to the E-frame.

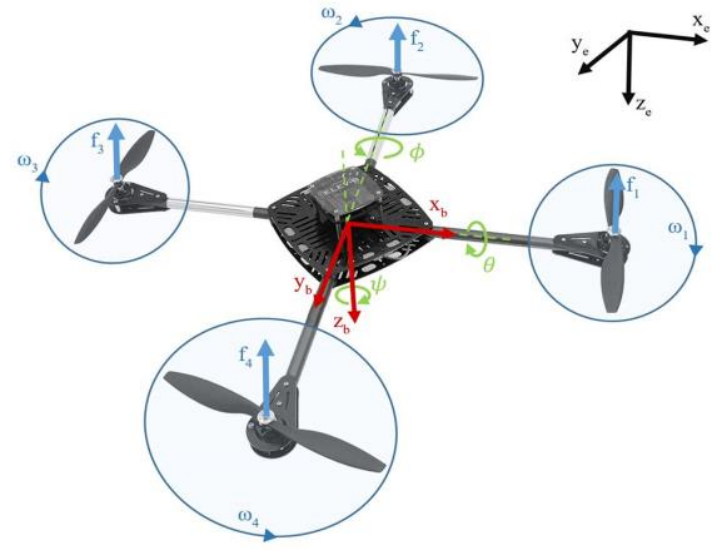

Figure 1: Multirotor system

The rotation matrix is used to map the orientation of a vector from the B-frame to E-frame [36].

$$
R=\left[\begin{array}{ccc}
c_{\theta} c_{\psi} & -c_{\phi} s_{\psi}+c_{\psi} s_{\phi} s_{\theta} & s_{\phi} s_{\psi}+c_{\phi} c_{\psi} s_{\theta} \\
c_{\theta} s_{\psi} & c_{\phi} c_{\psi}+s_{\phi} s_{\theta} s_{\psi} & -c_{\psi} s_{\phi}+c_{\phi} s_{\theta} s_{\psi} \\
-s_{\theta} & c_{\phi} s_{\theta} & c_{\phi} c_{\theta}
\end{array}\right]
$$

where $\mathrm{s}_{x}=\sin (x)$ and $c_{x}=\cos (x)$.

The below transfer matrix is also used to map the relation between the angular velocity $\left(\boldsymbol{\omega}=\left[\begin{array}{lll}\dot{\phi} & \dot{\theta} & \dot{\psi}\end{array}\right]^{T}\right)$ in the Bframe and Euler angles rates $(\dot{\boldsymbol{\Theta}})$ in the E-frame.

$$
\dot{\Theta}=\boldsymbol{T} \omega
$$

$$
\boldsymbol{T}=\left[\begin{array}{ccc}
1 & s_{\phi} t_{\theta} & c_{\phi} t_{\theta} \\
0 & c_{\phi} & -s_{\phi} \\
0 & s_{\phi} / c_{\theta} & c_{\phi} / c_{\theta}
\end{array}\right]
$$

where $t_{x}=\tan (x)$.

The state space dynamic model of the multirotor system can be derived using the Newton's second law [37]. The 6 DOF dynamic model including the attitude dynamics of the used multirotor system can be described as follow:

$$
\begin{gathered}
{\left[\begin{array}{c}
\ddot{x} \\
\ddot{y} \\
\ddot{z}
\end{array}\right]=\frac{1}{m}\left[\begin{array}{c}
s_{\psi} s_{\phi}+c_{\psi} s_{\theta} c_{\phi} \\
-c_{\psi} s_{\phi}+s_{\psi} s_{\theta} c_{\phi} \\
c_{\theta} c_{\phi}
\end{array}\right] T_{z}-\left[\begin{array}{l}
0 \\
0 \\
g
\end{array}\right]} \\
{\left[\begin{array}{c}
\ddot{\phi} \\
\ddot{\theta} \\
\ddot{\psi}
\end{array}\right]=\left[\begin{array}{c}
\left(\frac{I_{y}-I_{z}}{I_{x}}\right) \dot{\theta} \dot{\psi} \\
\left(\frac{I_{z}-I_{y}}{I_{y}}\right) \dot{\phi} \dot{\psi} \\
\left(\frac{I_{x}-I_{y}}{I_{z}}\right) \dot{\phi} \dot{\theta}
\end{array}\right]+\left[\begin{array}{c}
\frac{J_{p}}{I_{x}} \dot{\theta} \\
\frac{J_{p}}{I_{y}} \dot{\phi} \\
0
\end{array}\right] \Omega\left[\begin{array}{ccc}
\frac{1}{I_{x}} & 0 & 0 \\
0 & \frac{1}{I_{y}} & 0 \\
0 & 0 & \frac{1}{I_{z}}
\end{array}\right]\left[\begin{array}{c}
\tau_{\phi} \\
\tau_{\theta} \\
\tau_{\psi}
\end{array}\right]}
\end{gathered}
$$

where $\ddot{x}, \ddot{y}$ and $\ddot{z}$ define the linear acceleration in $x, y$ and $z$ axes in E-frame, respectively; $m$ denotes the vehicle's mass, and $g$ is the gravitational acceleration acting along the z-axis with respect to E-frame. $\ddot{\phi}, \ddot{\theta}$ and $\ddot{\psi}$ define the angular acceleration around $x, y$ and $z$ axes, respectively, with respect to E-frame. $J_{P}$ denotes the total rotational moment of inertia around the propeller axis. $I_{x}, I_{y}$ and $I_{z}$ are the moments of inertia along the $\mathrm{x}, \mathrm{y}$, and $\mathrm{z}$ axes. The inputs of the multirotor system $\tau_{\phi}, \tau_{\theta}$ and $\tau_{\psi}$ represent the input roll torque, pitch torque and yaw torque, respectively.

\section{B. Multilayer Feed-Forward Neural Networks}

A multilayer (FFNN) has been selected in this study to enhance the conventional state estimation technique. As shown in Fig. 2, a FFNN is comprised of one input layer, one or more hidden layers and one output layer.

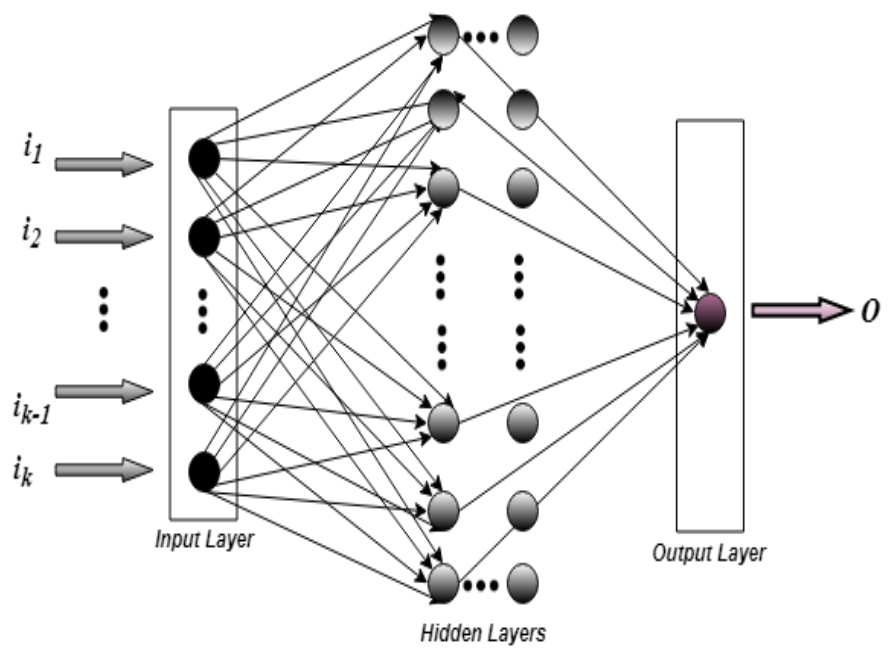

Figure 2: Structure of Multilayer Neural Network 
Each element of the input vector $I=\left[i_{1}, i_{2}, \ldots, i_{k}\right]$ is weighted by its corresponding weight of the weight matrix $W$ and the neuron bias $b$ is summed to produce the net input $n$.

$$
n=\sum_{j=1}^{k} w_{j} i_{j}+b
$$

Then an activation function $f$ is used to generate the neuron output $o$.

$$
o=f(n)
$$

In this study, a hyperbolic tangent activation function is used. It can be expressed as follows:

$$
f(x)=\frac{1-e^{-2 x}}{1+e^{-2 x}}
$$

\section{INTEGRATED DEEP LEARNING STATE ESTIMATION TECHNIQUE}

The UAV's dynamic states, namely velocity, position and attitude are measured using the onboard low cost IMU unit. Due to the immense inherent vibration of the multirotor's actuators, the measured orientation of the vehicle is noisy and inaccurate to a few degrees [38]. To better reject such noise sequences and enhance the performance of the attitude estimator, a precise integrated DL-based attitude estimation technique is developed. This section investigates the problem of precision attitude estimation using DNNs which are trained via modern techniques of deep learning.

\section{A. Kalman state estimation cycle}

The Kalman linear estimation technique is recognized as an optimal minimum mean square error (MMSE) state estimation method $[39,40]$. Such method estimates the unknown states based on recursive calculations lead to update the state estimate and its covariance as follows.

$$
\begin{gathered}
\hat{x}_{k+1 \mid K+1}=\hat{x}_{k+1 \mid K}+W_{K+1} v_{K+1} \\
P_{K+1 \mid K+1}=P_{K+1 \mid k}-W_{K+1} S_{K+1} W_{K+1}^{T}
\end{gathered}
$$

where $\hat{x}_{k+1 \mid K+1}$ and $P_{K+1 \mid K+1}$ denote the a posteriori state estimate and its covariance, respectively; $\hat{x}_{K+1 \mid K}$ and $P_{K+1 \mid K}$ represent the a priori state estimate and its covariance, respectively; the measurment innovation and the innovation covariance are denoted by $v_{K+1}$ and $S_{K+1}$, respectively; and $W_{K+1}$ denotes the optimal Kalman gain.

The linearized dynamic model of the quadrotor was used for the kalman filter design. The lineraization process was performed at hover point where the attitude states are around $0^{\circ}$. The linearzation of the quadrotor dynamics is thoroughly illustrated in [22]. The process and measurement noise covarince matrices of the filter are described in equations (1011). Selecting proper values for the diagonal variances is linked with accuracy of the dynamic model and measurments of the onborad sensors. In this study, the variances are tuned to ensure the convergence of the filter.

$$
Q_{k}=\left[\begin{array}{cccc}
q_{11} & 0 & \cdots & 0 \\
0 & q_{22} & & 0 \\
\vdots & & \ddots & \vdots \\
0 & 0 & \ldots & q_{66}
\end{array}\right]
$$

$$
R_{k}=\left[\begin{array}{cccc}
r_{11} & 0 & \cdots & 0 \\
0 & r_{22} & & 0 \\
\vdots & & \ddots & \vdots \\
0 & 0 & \cdots & r_{66}
\end{array}\right]
$$

\section{B. Deep learning-based training for FFNN}

Despite the effectiveness of the conventional Kalman estimation technique, a standalone Kalman state estimation does not show a great ability in eliminating the inherent noise sequences of the IMU measurements. This results in unstable hovering and more power consumed by actuators to compensate for these movements. To resolve this problem, DL techniques can be developed to identify representative models of the measurement noises and hence these noise levels can be further filtered out using robust learning method.

In this section, a Deep Neural Network (DNN) is designed and trained using modern DLNN technique to improve the quality of the Kalman attitude estimates. A dynamic model of the multirotor system is used to obtain the real dynamic response of the attitude states $X_{\text {real }}$.These values along with the Kalman state estimates are considered for the DNN state learning scheme. As shown in Fig. 3, the DNN uses the Kalman states estimates as inputs and the simulated real values are the target outputs while training the neural network. $U$ describes the control input vector to the multirotor vehicle.

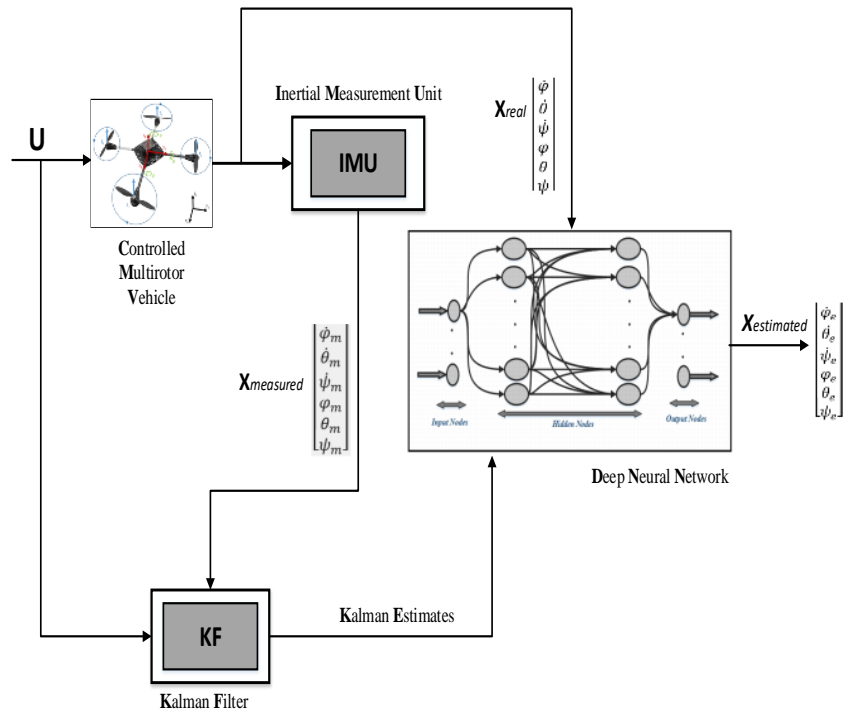

Figure 3: Simulation scheme of the proposed DNN state estimation.

The training process of the DNN is considered sensitive, and utilizing the conventional back propagation algorithm may 
yield an inaccurate performance of the neural network [25]. The back propagation algorithm experiences some problems associated with the training process of the DNN like computational load and overfitting, which can be tackled using some innovative training techniques of deep learning. For instance, the overfitting problem is resolved using Dropout learning method, which considers randomly selected nodes for training rather than the whole network as shown in Fig. 4 [41, 34].

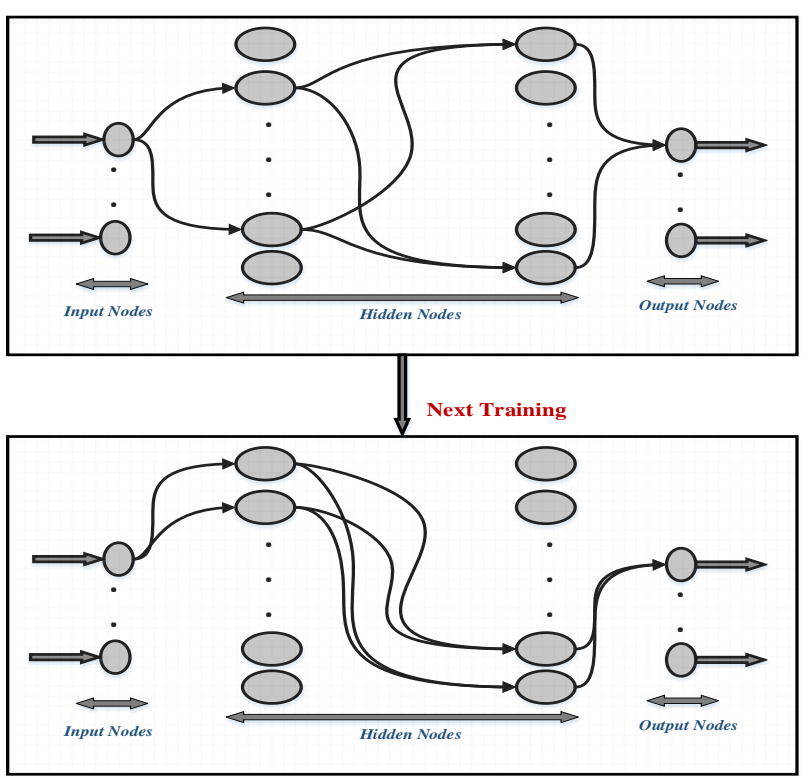

Figure 4: Dropout training technique

Dropout technique has proved its applicability for Bayesian Gaussian approximation purposes [35]. Considering the fact that Kalman filter is a special example of the Gaussian Processes (GPs) and deals with the Gaussian measurement's noise [42], the Dropout learning method has been implemented to have the 3-hidden-layers NN accurately trained to identify the unfiltered Gaussian noise associated with Kalman attitude estimates. A $20 \%$ of each hidden layer's nodes were randomly dropped out from training. In doing so, the overfitting problem is resolved, the computational complexity has been reduced and the remaining white noise has been accurately modelled and filtered out. In this study, a dropout-based LevenbergMarquardt training technique is developed.

The Levenberg-Marquardt technique is used where FFNN weights are tuned based on the minimization of the Mean Square Error (MSE) [43]:

$$
M S E=\frac{1}{2 \mathrm{n}} \sum_{k=1}^{n}\left(Y_{k}-\widehat{Y}_{k}\right)^{2}=\frac{1}{2 \mathrm{n}} \sum_{k=1}^{n} e^{2}
$$

where $Y_{k}$ and $\widehat{Y}_{k}$ are the target and evaluated network outputs; respectively, $\mathrm{n}$ represents the number of training data points. The Hessian matrix is approximated as:

$$
H=\mathrm{J}(\nabla \mathrm{MSE})=J^{T} J
$$

where $\mathbf{J}$ is the Jacobian matrix which comprises the error derivatives with respect to the tuned weights and biases. The gradient function can be computed as:

$$
\delta=\frac{\partial M S E}{\partial w}=J^{T} e
$$

The network weights parameters are optimized via the backward path of the network, as:

$$
\begin{gathered}
w_{k+1}=w_{k}+\Delta w \\
\Delta w=-(H+\mu I)^{-1} \delta
\end{gathered}
$$

where $\mu$ defines the adaptive learning rate and $I$ defines the identity matrix.

Fig. 5 illustrates the proposed incorporation of the dropout learning technique in the Levenberg-Marquardt training cycle. As seen, two sets of network inputs are considered in learning process. $S_{1}$ represents the noisy Kalman attitude estimates while $S_{2}$ represents the real attitude states. The training of the DNN is performed in two stages; the first one uses the forward path to compute the errors and the second stage updates the network weights using the gradient function $\frac{\partial M S E}{\partial w}$. The dropout technique is implemented to exclude a ratio of the layer nodes randomly from the training cycle.

Fig. 6 illustrates the performance of the validation of the obtained DNN model. The model results shows an excellent agreement with the training data, validation data, testing data and the whole data.

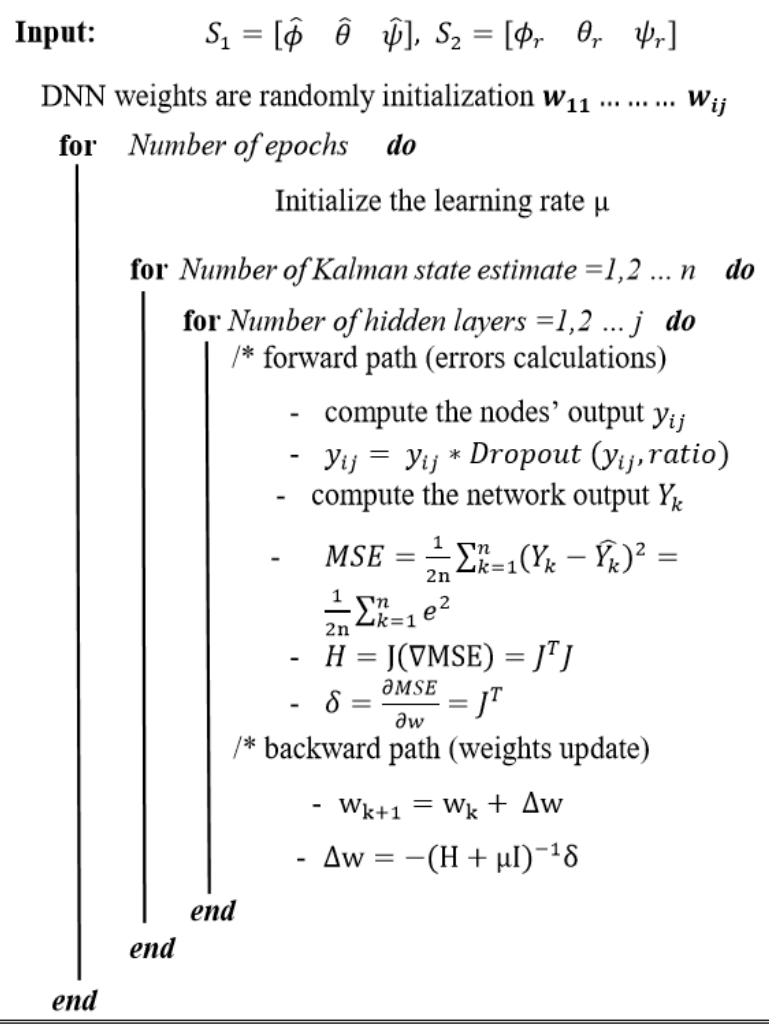

Figure 5: Dropout method for attitude state estimation 


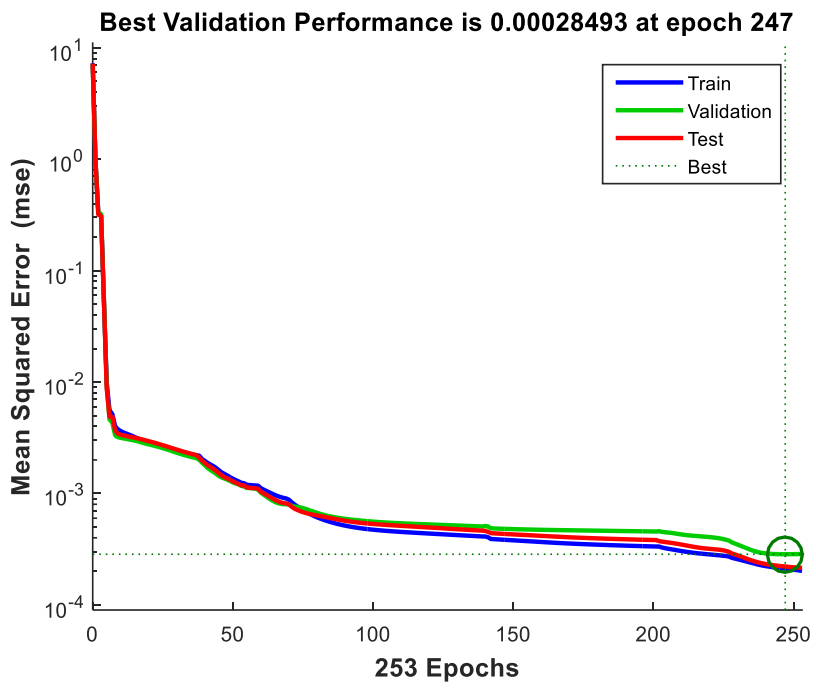

Figure 6: The Mean Squared Error (MSE) for the DNN model.

Fig. 7 illustrates the regression performances of the FFNN mode. From the regression plots, it can be concluded that the FFNN model has exhibited an excellent matching with the training data, validation data, testing data and the whole data.
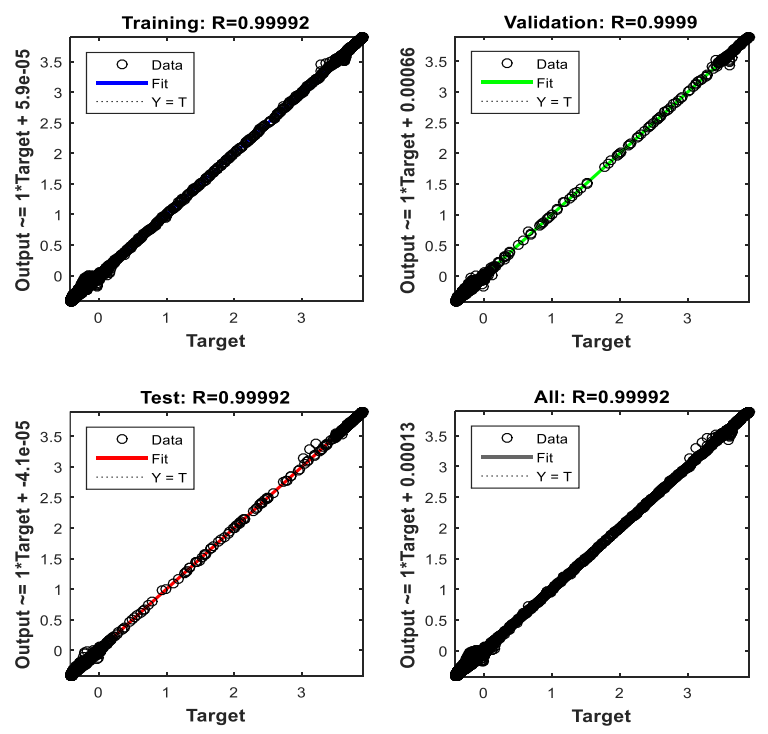

Figure 7: Regression performances for the obtained FFNN model.

\section{Simulation}

This section illustrates the simulation environment which was used to demonstrate the performance of the proposed attitude estimation technique. The IMU attitude measurements were modelled by adding white noise sequences to the real states acquired from the multirotor model. The proposed DLNN sensor fusion technique takes the attitude real states of the multirotor and the estimates states as targets and inputs, respectively, to train the DNN. The Dropout method was used to ensure no overfitting and make the training less computational as random nodes are considered for the network training. To demonstrate the performance of the DLNN state estimation approach, a near hover simulation flight test was conducted where the multirotor is commanded to change its heading within few degrees and the roll and pitch angles are somewhat around zero. Under Gaussian noise assumptions, it is expected that the Kalman filter achieves its optimal state estimation. The results of the proposed DLNN state estimation have been compared to the conventional Kalman filter to prove the attained enhancement in attitude estimation. The presented attitude results illustrate the enhancement in attitude estimation accomplished by the Deep-Learning-Based Neural Network (DLNN). Furthermore, a comparison study of different state estimation techniques is addressed to demonstrate the performance of the proposed method against other robust adaptive state estimation techniques.

\section{A. Attitude estimation}

Figures 8-10 present the roll, pitch and yaw angles state estimation. The figures exhibit the signals of the noisy IMU measurements, Kalman estimate, DLNN state estimates and the real states acquired from the simulation model of the multirotor. Fig. 8 shows the conventional and the DLNN roll angle estimation. It can be noticed that the DLNN has shown a better noise rejection compared to the KF. The KF has a Root Mean Square Error of Estimation (RMSEE) of 0.2738 while the error is 0.0286 for the DLNN approach. The pitch angle state estimation is demonstrated in Fig. 9. The DLNN approach has predicted the unfiltered white noise and filtered it out through the simulation. Moreover, the DLNN learning approach has captured the coupling dynamics between the attitude states (see the zoomed portion of the figure). The pitch angle is estimated with RMSEE values of 0.2711 and 0.0627 for the KF and the DLNN, respectively.

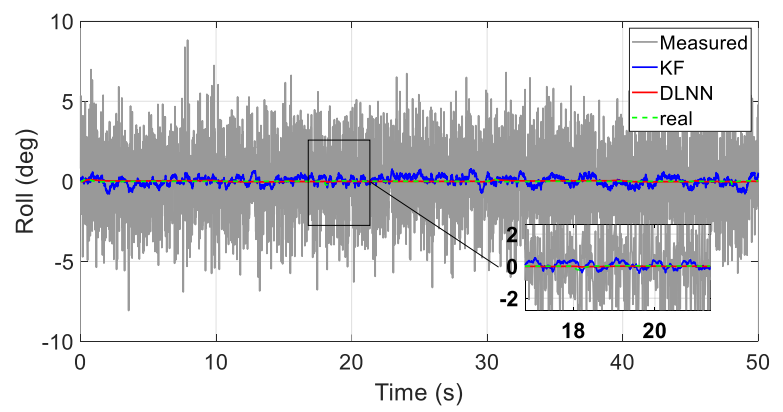

Figure 8: Roll angle state estimation

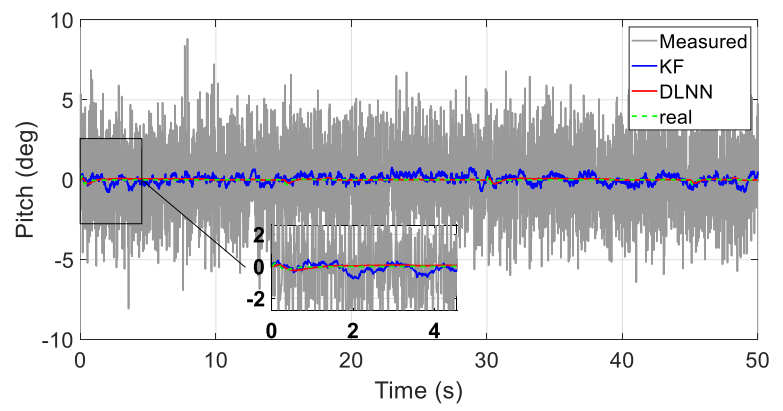

Figure 9: Pitch angle state estimation

Fig. 10 represents the yaw angle state estimation. The multirotor is commanded to change its heading by few degrees. 
It is clear from the Figure that DLNN is performing better in terms of coincidence with the real state. The yaw angle is estimated with an RMSEE error of 0.3173 for the KF and 0.2355 for the DL. Fig. 11 illustrates the estimation error of the attitude angles. As can be observed, the DLNN smaller amount of estimation errors throughout the flight.

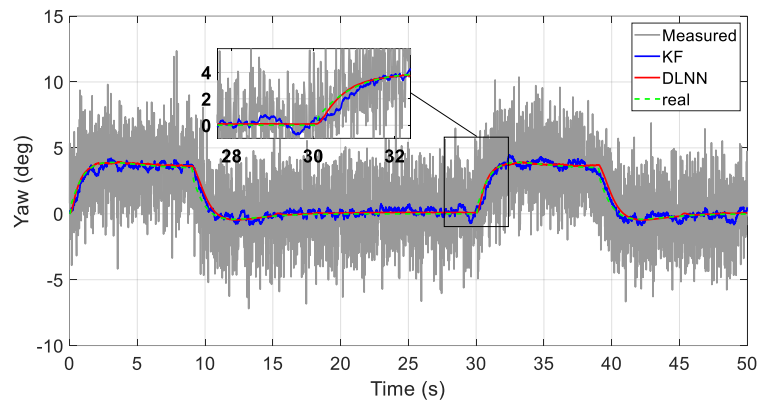

Figure 10: Yaw angle state estimation
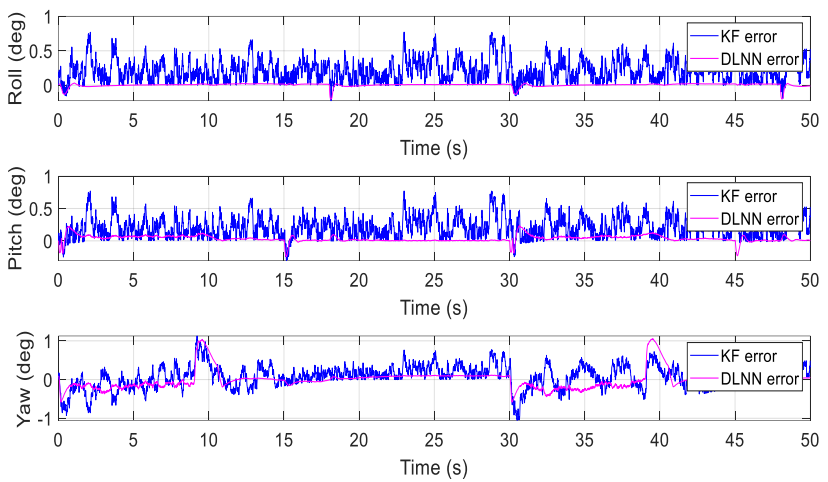

Figure 11: Attitude states estimation errors

The attitude rates estimation results are illustrated below in Figures 12-14. The DLNN state estimation method results demonstrate an accurate state estimation in terms of rejecting the added noise and coincidence with the real angular states.

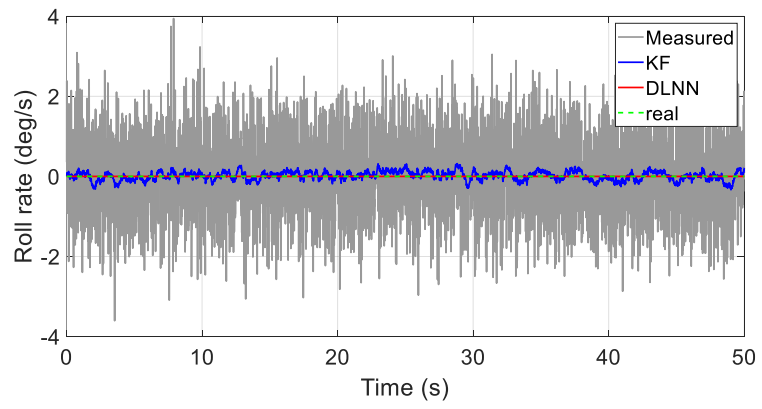

Figure 12: Roll rate state estimation.

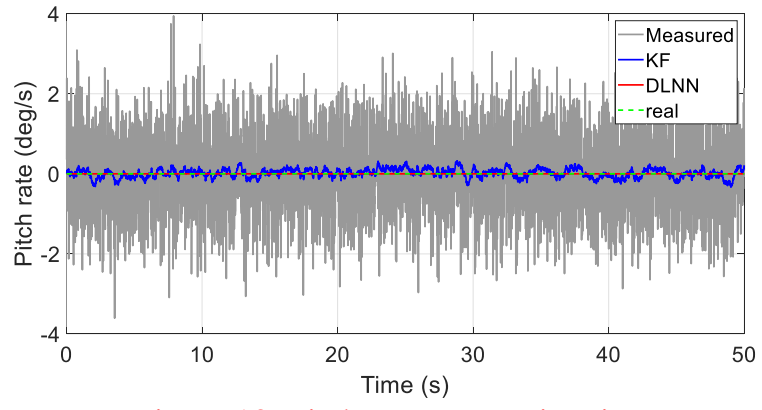

Figure 13: Pitch rate state estimation

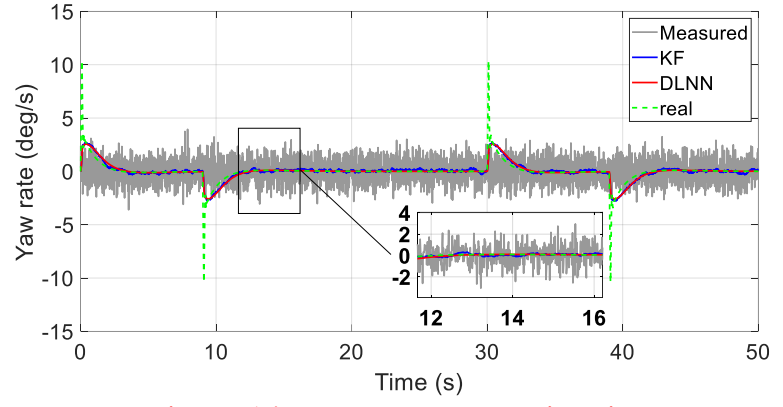

Figure 14: Yaw rate state estimation.

\section{B. Comparison study of state estimation techniques}

This section presents a comparison study of different state estimation techniques in order to demonstrate the performance of the proposed method against other robust conventional and adaptive state estimation techniques. An Adaptive Fuzzy Kalman Filter (AFKF) has been developed to compare the performance of the proposed DLNN method with the adaptive state estimation. The AFKF approach adopts the covariancematching algorithm to evaluate the degree of matching between the actual covariance of the of the innovation sequence of the filter with its theoretical value [44]. Based on the degree of matching, an adjustment of the measurement noise matrix is computed at each state estimation cycle using a Fuzzy Inference System (FIS).

Figure 15 shows the heading state estimation using conventional, adaptive and Deep-Learning based estimation techniques. From the Figure, it can be concluded that the AFKF shows faster convergence to the real value of the state than KF. However, in terms of noise reduction both AFKF and KF show similar behavior throughout the experiment. On the other side, DLNN shows a significant capability of noise reduction and better coincidence with the real heading state.

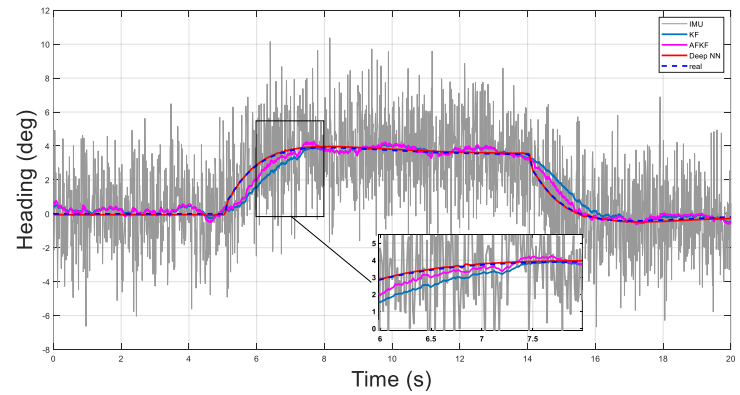

Figure 15: Heading state estimation using different state estimation techniques

\section{EXPERIMENTAL VALIDATION}

In real flight scenarios, it is not expected that the characteristics of measurement noise are Gaussian distributed. This makes the optimal state estimation not guaranteed unless accurate tuning of the filter's parameters and training for the DNN is made. A fine training for the proposed DNN has been conducted using collected real flight data. A real hover flight was carried out to validate the performance of the proposed integrated state estimation technique. A multirotor equipped 
with PX4 autopilot and Jetson tx1 onboard computer is used with Optitrack indoor tracking system. The exact drone's location in $\mathrm{x}, \mathrm{y}$ and $\mathrm{z}$ is sent by the Optitrack system which has position accuracy of $0.76 \mathrm{~mm}$. The Optitrack tracking system runs motive software for motion capture and live streaming. The tracking system works by tracking the markers that are placed on the drone's body using 16 cameras distributed evenly in the room as shown in Fig. 16. The mocap ros package which is running on the on-board computer takes the data from the Optitrack system and passes it to the autopilot to hold the drone at specific location. The pose topic that is received from the Optitrack system is with a frequency of 120 messages/second and the pose topic recorded by the autopilot and sent is with 30 messages/second.

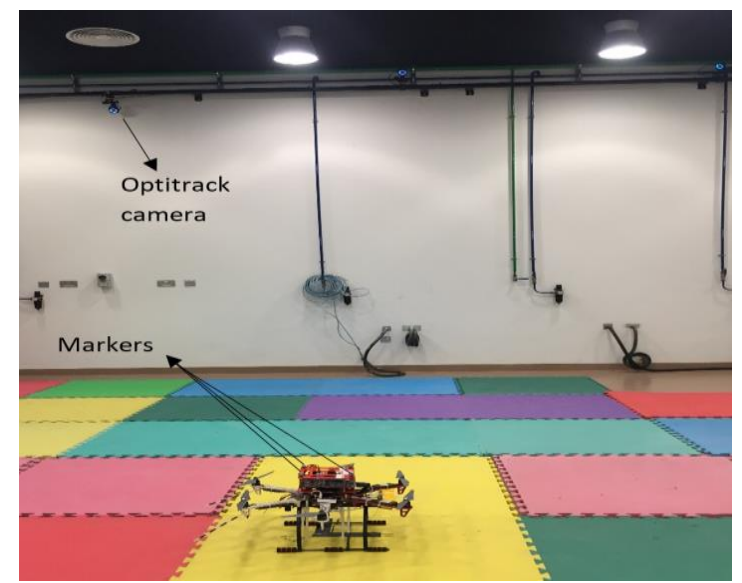

Figure 16: Multirotor platform and the Optitrack system

The data received from the Optitrack system is compared to the data received from the autopilot to correct the drone's position in a closed loop system. Fig. 17 below shows the drone flying in position hold mode.

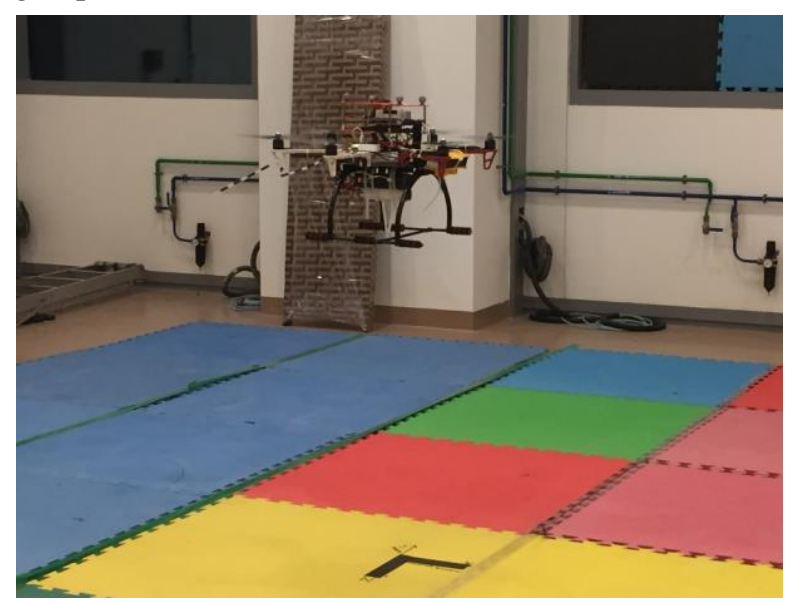

Figure 17: Multirotor at hover.

The advantage of using the PX4 autopilot is that its estimation and Control Library (ECL) provides IMU state estimates which are based on implementing the Kalman estimation techniques. As these estimates were provided in the quaternion format, a conversion to the Euler format was done to get the Euler angles. In this real hover test, the integrated
DLNN sensor fusion technique has been applied to estimate the attitude states of the multirotor system. The attitude angles are expected to have small values around zero degrees. In such real flight tests, the exact real states values cannot be directly determined. However, to evaluate the performance of the proposed sensor fusion method, the estimation results are compared to the precise attitude values captured by the Optitrack system.

Figures 18-20 show the attitude estimation results. From the figures, it can be discerned that the proposed DLNN estimation has displayed better agreement with the Optitrack values. Moreover, the integrated DLNN approach has demonstrated its capability in reducing the real unpredictable measurements' noise and bias associated with the onboard IMU unit.

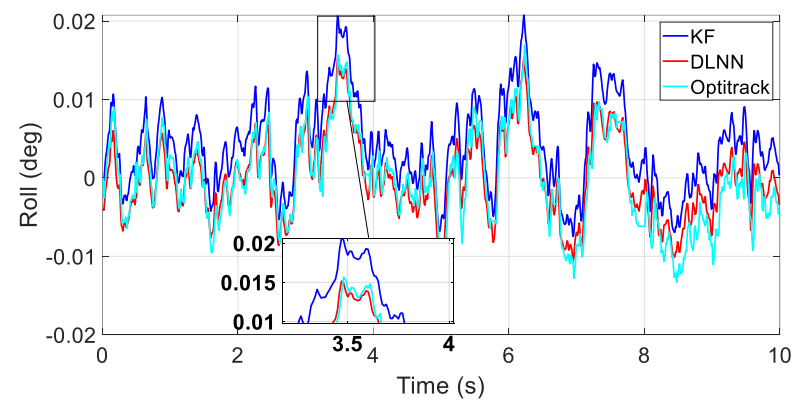

Figure 18: Roll angle state estimation

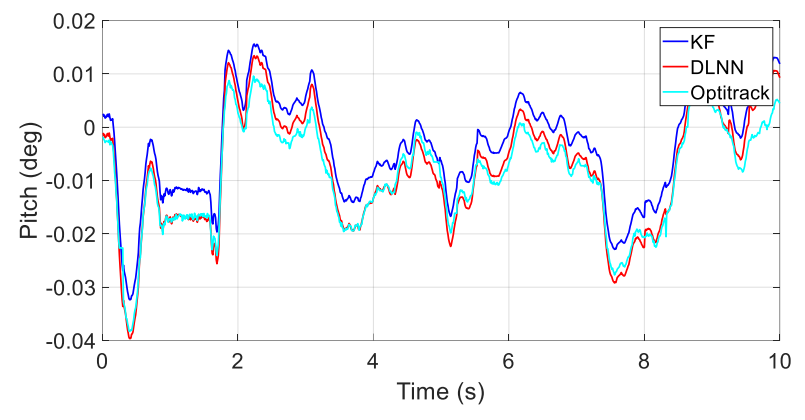

Figure 19: Pitch angle state estimation

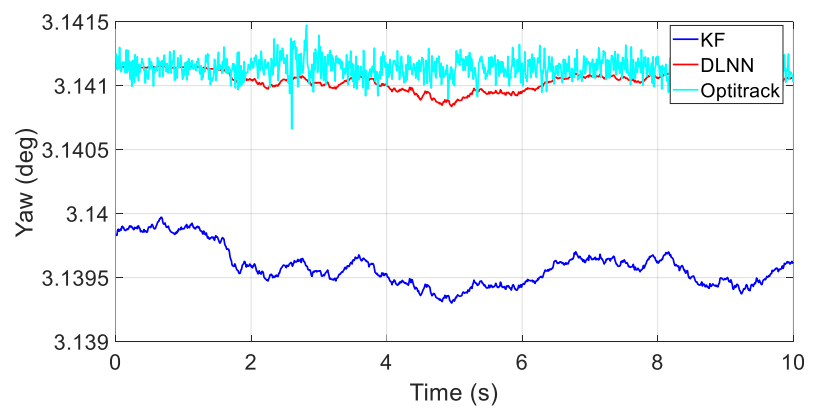

Figure 20: Yaw angle state estimation

Table 2 concludes the Root Mean Squared Errors in Estimation (RMSEE) of both standalone KF and DLNN in attitude estimation. It is discerned that the pitch and roll states of the quadrotor are accurately estimated and enhanced. The heading state estimation is also significantly improved using the DLNN based state estimation approach. 
Table 2: RMSEE values for KF and DLNN

\begin{tabular}{cll}
\hline \hline State & KF RMSEE & DLNN RMSEE \\
\hline$\phi$ & 0.0017 & 0.00016 \\
$\theta$ & 0.0018 & 0.00023 \\
$\psi$ & 0.0130 & 0.00200 \\
\hline
\end{tabular}

\section{CONCLUSION}

Deep learning methods are data-driven algorithms use their complex nonlinear transformations which generated using the nonlinear input-output mappings to identify the most representative models for the uncertainty in data. This study has employed these algorithms to enhance the accuracy of the state estimation. The proposed state estimation technique is based on training the DNN using the dropout method to ensure identifying representative, fitted and robust models for the unfiltered measurement's noise. This novel technique was tested using a simulation environment and an actual experimental setup. The DLNN estimation results demonstrates a significant ability in identifying the noise models associated with the Kalman estimates. Moreover, the proposed DLNN attitude estimation results exhibit superiority over the conventional attitude estimates.

\section{REFERENCES}

[1] M. Al-Sharman, M. A. Al-Jarrah and M. Abdel-Hafez, "Auto Takeoff and Precision Terminal-Phase Landing using an Experimental Optical Flow Model for GPS/INS Enhancement," ASCE-ASME Journal of Risk and Uncertainty in Engineering Systems, Part B: Mechanical Engineering, vol. 4, no. 2, 2018.

[2] S. Grzonka, G. Grisetti and W. Burgard, "A Fully Autonomous Indoor Quadrotor," IEEE Transactions on Robotics, vol. 28, no. 1, pp. 90 - 100, 2012.

[3] P. Serra, R. Cunha, T. Hamel, D. Cabecinhas and C. Silvestre, "Landing of a Quadrotor on a Moving Target Using Dynamic Image-Based Visual Servo Control," IEEE Transactions on Robotics, vol. 32, no. 6, pp. 1524$1535,2016$.

[4] L. F. Luque-Vega , B. Castillo-Toledo , A. Loukianov and L. E. Gonzalez-Jimenez, "Power line inspection via an unmanned aerial system based on the quadrotor helicopter," in MELECON 2014 - 2014 17th IEEE Mediterranean Electrotechnical Conference, Lebanon, 2014.

[5] D. Zheng, H. Wang, W. Chen and Y. Wang, "Planning and Tracking in Image Space for Image-Based Visual Servoing of a Quadrotor," IEEE TRANSACTIONS ON INDUSTRIAL ELECTRONICS, vol. 65, no. 4, pp. 3376-3388, 2018.

[6] J. Thomas, M. Pope, G. Loianno, E. W. Hawkes, M. A. Estrada, H. Jiang, M. R. Cutkosky and V. Kumar, "Aggressive Flight With Quadrotors for Perching on Inclined Surfaces," Journal of Mechanisms and Robotics, vol. 8 , no. 5,2016

[7] G. Loianno, C. Brunner, G. McGrath and V. Kumar, "Estimation, Control, and Planning for Aggressive Flight With a Small Quadrotor With a Single Camera and IMU," IEEE Robotics and Automation Letters, vol. 2, no. 2, pp. 404 - 4011, 2017.

[8] R. Sanz, L. Ródenas, P. García and P. Castillo, "Improving attitude estimation using inertial sensors for quadrotor control systems," in International Conference on Unmanned Aircraft Systems, Florida, 2014.

[9] L. Chang, J. Li and S. Chen, "Initial Alignment by Attitude Estimation for Strapdown Inertial Navigation Systems," IEEE Transactions on Instrumentation and Measurement, vol. 64, no. 3, pp. 784 - 794, 2015.
[10] P. Marantos, Y. Koveos and K. J. Kyriakopoulos, "UAV State Estimation Using Adaptive Complementary Filters," IEEE Transactions on Control Systems Technology, vol. 24, no. 4, pp. 1214-1226, 2016.

[11] H. Chao, C. Coopmans, L. Di and Y. Chen, "Comparative Evaluation of Low-Cost IMUs for Unmanned Autonomous Systems," in IEEE International Conference on Multisensor Fusion and Integration for Intelligent Systems, Utah, 2010.

[12] M. Ghobadi, P. Singla and E. T. Esfahani, "Robust Attitude Estimation from Uncertain Observations of Inertial Sensors Using Covariance Inflated Multiplicative Extended Kalman Filter," IEEE TRANSACTIONS ON INSTRUMENTATION AND MEASUREMENT, vol. 67, no. 1, pp. 209-217, 2018

[13] Y. Zhao, "Cubature + Extended Hybrid Kalman Filtering Method and Its Application in PPP/IMU Tightly Coupled Navigation Systems," IEEE Sensors Journal, vol. 15, no. 12, pp. 6973 - 6985, 2015.

[14] M. Al-Sharman, M. Abdel-Hafez and M. Al-Omari, "Attitude and Flapping Angles Estimation for a Small-Scale Flybarless Helicopter Using a Kalman Filter," IEEE sensors Journal , vol. 15, no. 4, pp. 2114 $-2122,2015$.

[15] . A. Battiston, I. Sharf and M. Nahon, "Attitude estimation for normal flight and collision recovery of a quadrotor UAV," in International Conference on Unmanned Aircraft Systems (ICUAS), Florida, 2017.

[16] T.-A. Johansen and R. Kristiansen, "Quadrotor attitude estimation using adaptive fading multiplicative EKF," in American Control Conference (ACC), 2017, Washington, 2017.

[17] M. Tarhan and E. Altuğ, "EKF Based Attitude Estimation and Stabilization of a Quadrotor UAV Using Vanishing Points in Catadioptric Images," Journal of Intelligent \& Robotic Systems, vol. 62, no. 3-4, pp. 587-607, 2011.

[18] J. Shin , K. Kwak, S. Kim and H. J. Kim, "Adaptive range estimation in perspective vision system using neural networks," IEEE/ASME Transactions on Mechatronics, 2018.

[19] S. Yin , . X. Zhu, J. Qiu and H. Gao, "State Estimation in Nonlinear System Using Sequential Evolutionary Filter," IEEE Transactions on Industrial Electronics, vol. 63 , no. 6, pp. 3786-3794, 2016.

[20] M. A. K. Jaradat and M. F. Abdel-Hafez, "Enhanced, delay dependent, intelligent fusion for INS/GPS navigation system," IEEE Sensors Journal, vol. 14, no. 5, pp. 1545-1554, 2014.

[21] C.-L. Lin , . Y.-M. Chang, C.-C. Hung, C.-D. Tu and C.-Y. Chuang, "Position Estimation and Smooth Tracking With a Fuzzy-Logic-Based Adaptive Strong Tracking Kalman Filter for Capacitive Touch Panels," IEEE Transactions on Industrial Electronics, vol. 62, no. 8, pp. 5097 5108, 2015.

[22] M. Al-Sharman , B. J. Emran, M. A. Jaradat, H. Najjaran, R. Al-Husari and Y. Zweiri, "Precision Landing Using an Adaptive Fuzzy MultiSensor Data Fusion Architecture," Applied Soft Computing , 2018.

[23] M. N. Ibarra-Bonilla, P. J. Escamilla-Ambrosio and J. M. RamirezCortes, "Attitude estimation using a Neuro-Fuzzy tuning based adaptive Kalman filter," Journal of Intelligent \& Fuzzy Systems, vol. 29, no. 2, pp. 479-488, 2015.

[24] M. A. K. Jaradat and M. F. Abdel-hafez, "Non-linear autoregressive delay-dependent INS/GPS navigation system using neural networks," IEEE sensors journal, vol. 17, no. 5, pp. 1105-1115, 2017.

[25] Y. LeCun, Y. Bengio and G. Hinton, "Deep Learning," Nature, vol. 521, pp. 444-436, 2015.

[26] O. Costilla-Reyes, P. Scully and K. B. Ozanyan, "Deep Neural Networks for Learning Spatio-Temporal Features From Tomography Sensors," IEEE TRANSACTIONS ON INDUSTRIAL ELECTRONICS, vol. 65, no. 1, pp. 645-63, 2018.

[27] F.-C. Chen and M. R. Jahanshahi, "NB-CNN: Deep Learning-Based Crack Detection Using Convolutional Neural Network and Na" ive Bayes Data Fusion," IEEE TRANSACTIONS ON INDUSTRIAL ELECTRONICS, vol. 65, no. 5, pp. 4392-4400, 2018.

[28] J. Lei, . Q. Liu and X. Wang, "Deep Learning-Based Inversion Method for Imaging Problems in Electrical Capacitance Tomography," IEEE 
Transactions on Instrumentation and Measurement, vol. 67, no. 9, pp. $2107-2118,2018$.

[29] J. Sun , C. Yan and J. Wen, "Intelligent Bearing Fault Diagnosis Method Combining Compressed Data Acquisition and Deep Learning," IEEE Transactions on Instrumentation and Measurement, vol. 67, no. 1, pp. 185 - 195, 2018.

[30] K. Cheon, . J. Kim, M. Hamadache and D. Lee, "On Replacing PID Controller with Deep Learning Controller for DC Motor System," Journal of Automation and Control Engineering, vol. 3, no. 6, 2015.

[31] A. Carrio, C. Sampedro, A. Rodriguez-Ramos and P. Campoy, "A Review of Deep Learning Methods and Applications for Unmanned Aerial Vehicles," Journal of Sensors, pp. 1-13, 2017.

[32] M. Merida-Floriano, F. Caballero, D. Garc'ia-Morales , F. Casares and . L. Merino, "Bioinspired Vision-only UAV Attitude Rate Estimation using Machine," in International Conference on Unmanned Aircraft Systems (ICUAS), Florida, 2017.

[33] R. G. Krishnan, U. Shalit and D. Sontag, "Deep Kalman filters," in NIPS 2016 Workshop: Advances in Approximate Bayesian Inference, 2016.

[34] N. Srivastava, G. Hinton, A. Krizhevsky, I. Sutskever and R. Salakhutdinov, "Dropout: A Simple Way to Prevent Neural Networks from," Journal of Machine Learning Research, vol. 15, no. 1, pp. 19291958, 2014.

[35] Y. Gal and Z. Ghahramani, "Dropout as a Bayesian Approximation: Representing Model Uncertainty in Deep Learning," in international conference on machine learning, New York, 2016.

[36] E. G. James, Matrix Algebra Theory, Computations, and Applications in Statistics, springer, 2007.

[37] R. Mahony , V. Kumar and P. Corke , "Multirotor Aerial Vehicles: Modeling, Estimation, And Control of Quadrotor," IEEE Robotics \& Automation Magazine, vol. 19, no. 3, pp. 20-32, 2012.

[38] H. Chao, C. Coopmans and Y. Chen, "A Comparative Evaluation of Low-Cost IMUs for Unmanned Autonomous Systems," in IEEE international conference on Multisensor Fusion and Integration for Intelligent Systems , Utah, 2010.

[39] Z.-Q. Zhang, X.-L. Meng and J.-K. Wu, "Quaternion-Based Kalman Filter With Vector Selection for Accurate Orientation Tracking," IEEE Transactions on Instrumentation and Measurement, vol. 61, no. 10, pp. 2817 - 2824, 2012.

[40] M. Ghobadi, P. Singla and E. T. Esfahani , "Robust Attitude Estimation from Uncertain Observations of Inertial Sensors Using Covariance Inflated Multiplicative Extended Kalman Filter," IEEE Transactions on Instrumentation and Measurement, vol. 67, no. 1, pp. 209 - 217, 2018.

[41] G. E. Dahl, T. . N. Sainath and G. E. Hinton, "Improving deep neural networks for LVCSR using rectified linear units and dropout," in IEEE International Conference on Acoustics, Speech and Signal Processing, Vancouver, BC, 2013.

[42] S. Reece and S. Roberts, "An introduction to Gaussian processes for the Kalman filter expert," in 13th International Conference on Information Fusion, Edinburgh, 210.

[43] . J. C. Principe, N. R. Euliano and W. C. Lefebvre, Neural and Adaptive Systems: Fundamentals through Simulations, New York : Wiley, 2000 .

[44] M. K. Al-Sharman, M. A. Jaradat and M. F. Abdel-Hafez, "Intelligent attitude and flapping angles estimation of flybarless helicopters under near-hover conditions," Journal of the Franklin Institute, vol. 355, no. 14, pp. 6830-6858, 2018.

[45] V. N. Nguyen, R. Jenssen and D. Roverso, "Automatic autonomous vision-based power line inspection: A review of current status and the potential role of deep learning," International Journal of Electrical Power \& Energy Systems, vol. 99, pp. 107-120, 2018.

[46] H. Yang , B. Hu and L. Wang, "A Deep Learning based Handover Mechanism for UAV Networks," in The 20th International Symposium on Wireless Personal Multimedia Communications, Bali, 2018.

[47] A. Maher, C. Li, H. Hu and B. Zhang, "Realtime Human-UAV Interaction Using Deep Learning," in Chinese Conference on Biometric Recognition, Beijing, 2017.
[48] . C. Sheppard and M. Rahnemoonfar, "Real-time scene understanding for UAV imagery based on deep convolutional neural networks," in IEEE International Geoscience and Remote Sensing Symposium (IGARSS), Texas , 2017.

[49] . Z. Fan, J. Lu , M. Gong, H. Xie and . E. D. Goodman , "Automatic Tobacco Plant Detection in UAV Images via Deep Neural Networks," IEEE Journal of Selected Topics in Applied Earth Observations and Remote Sensing, vol. 11, no. 3, pp. 876-887, 2018.

[50] J.-S. Zhang , J. Cao and B. Mao, "Application of deep learning and unmanned aerial vehicle technology in traffic flow monitoring," in International Conference on Machine Learning and Cybernetics, Ningbo, 2017.

[51] "Perception, Guidance, and Navigation for Indoor Autonomous Drone Racing Using Deep Learning," IEEE ROBOTICS AND AUTOMATION LETTERS, vol. 3, no. 3, pp. 2539-2544, 2018.

[52] A. Zeggada, F. Melgani and Y. Bazi , "A Deep Learning Approach to UAV Image Multilabeling," IEEE Geoscience and Remote Sensing Letters, vol. 14, no. 5, pp. 694-698, 2017.

[53] N. Ammour, H. Alhichri, Y. Bazi, B. Benjdira, N. Alajlan and M. Zuair, "Deep Learning Approach for Car Detection in UAV Imagery," Remote Sensing, vol. 9, no. 4, 2017.

[54] H. Yao, Q. Yu, X. Xing, F. He and . J. Ma, "Deep-learning-based Moving Target Detection for Unmanned Air," in 36th Chinese Control Conference (CCC), Dalian, 2017.

[55] D. Cisek, M. Mahajan , J. Dale, S. Pepper, Y. Lin and S. Yoo, "A Transfer Learning approach to parking lot," in New York Scientific Data Summit (NYSDS), New York, 2017.

[56] N. Smolyanskiy, A. Kamenev, J. Smith and S. Birchfield, "Toward LowFlying Autonomous MAV Trail Navigation using Deep Neural Networks for Environmental Awareness," in IEEE/RSJ International Conference on Intelligent Robots and Systems (IROS), Vancouver, 2017. 